\title{
Meta
}

Journal des traducteurs

Translators' Journal

Masao KUNIHIRO, Sen NISHIYAMA et Nobuo KANAYAMA

(1969): TSUYAKU : Eigokaiwa kara dojitsuyaku mode

(L'Interprétation : de la conversation anglaise à

l'interprétation simultanée), Tokyo, Nihonhososhuppankyokai

\section{Daniel Gile}

Volume 35, numéro 2, juin 1990

URI : https://id.erudit.org/iderudit/002901ar

DOI : https://doi.org/10.7202/002901ar

Aller au sommaire du numéro

Éditeur(s)

Les Presses de l'Université de Montréal

ISSN

0026-0452 (imprimé)

1492-1421 (numérique)

Découvrir la revue

Citer ce compte rendu

Gile, D. (1990). Compte rendu de [Masao KUNIHIRO, Sen NISHIYAMA et Nobuo

KANAYAMA (1969): TSUYAKU : Eigokaiwa kara dojitsuyaku mode

(L'Interprétation : de la conversation anglaise à l'interprétation simultanée),

Tokyo, Nihonhososhuppankyokai]. Meta, 35(2), 429-432.

https://doi.org/10.7202/002901ar d'utilisation que vous pouvez consulter en ligne. 
Masao KUNIHIRO, Sen NISHIYAMA et Nobuo KANAYAMA (1969): TSUYAKU: Eigokaiwa kara dojitsuyaku made (L'Interprétation: de la conversation anglaise à l' interprétation simultanée), Tokyo, Nihonhososhuppankyokai.

Ce livre, dont la première édition date de novembre 1969 et la sixième et dernière édition d'octobre 1970, qui est maintenant épuisé et introuvable dans les librairies de Tokyo, est probablement le meilleur parmi la dizaine d'ouvrages publiés au Japon sur l'interprétation entre 1961 et 1985.

Contrairement à ce que semble indiquer son titre, il s'agit non pas d'un manuel scolaire devant mener l'étudiant du perfectionnement en anglais à la simultanée, mais d'une tentative de présentation générale de l'interprétation de conférence, analogue à celle du Manuel de l'interprète de Jean Herbert, ou de l'Interprète dans les conférences internationales de D. Seleskovitch.

Ses trois coauteurs sont d'anciens interprètes (qui ne sont plus actifs) ayant chacun une deuxième, voire une troisième spécialité: Masao KUNIHIRO est également ethnologue, mais est surtout connu comme professeur d'anglais; Nishiyama SEN, ingénieur et cadre chez SONY, est devenu célèbre à l'occasion des missions «Apollo» américaines qu'il a interprétées en direct à la télévision japonaise - et qui ont suscité une grande vague de popularité de l'interprétation à la fin des années 1960; Nobuo KANAYAMA, qui est l'auteur de l'idée de ce livre, est également spécialiste de gestion.

Cet ouvrage de 230 pages s'articule en 21 chapitres et trois parties. La première, consacrée à la «théorie», relève plutôt de la vulgarisation et énonce des principes méthodologiques de la communication et de l'interprétation sans référence à de véritables théories ou à des travaux de recherche. Elle présente notamment le schéma, devenu classique sous différentes variantes, de l'interprète comme décodeur-encodeur intermédiaire dans la communication entre un émetteur-encodeur et un destinataire-décodeur, évoque les conditions nécessaires à une interprétation de bonne qualité, délimite les différents types d'interprétation, précise la qualification professionnelle de l'interprète, et parle longuement des problèmes de langue.

La deuxième partie porte sur la formation. Elle commence par la présentation sommaire de quelques idées sur les principes d'élocution applicables aux interprètes et du 
langage de la procédure, passe aux principes de l'interprétation consécutive et de la prise de notes, puis à la simultanée, pour terminer par de nouvelles considérations sur la langue, et notamment sur l'enrichissement du vocabulaire nécessaire à l'interprétation.

La troisième partie, intitulée «la pratique», porte sur le comportement de l'interprète face à ses auditeurs, sur des questions de déontologie, d'hygiène de la vie, et sur les installations techniques de la simultanée.

Dans l'ensemble, un contenu très varié, et impossible à traiter en profondeur en moins de 250 pages. Toutefois, en tant que manuel, le livre contient beaucoup d'idées, et surtout des idées dont les Occidentaux se détournent pour la plupart.

En premier lieu, les auteurs notent et soulignent l'existence de limites à la «capacité d'interprétation», ainsi que la responsabilité de l'orateur, qui doit être conscient du fait que son message doit passer par un canal de transmission ayant des dimensions finies et en tenir compte. Ce point de vue nous semble plus réaliste que celui adopté par bien des collègues occidentaux, qui cherchent à faire de l'interprète l'égal intellectuel de tout orateur et qui lui attribuent une compréhension du discours bien supérieure à sa modeste et superficielle réalité.

Dans le même ordre d'idée, les auteurs soulignent que la communication directe entre émetteur et destinataire est accompagnée d'une certaine perte, et que dans la communication passant par l'interprète, la perte entre émetteur et interprète s'ajoute à la perte entre interprète et destinataire. Ils soulignent aussi qu'un bon interprète peut être plus clair et plus logique dans son expression que son client japonais - on peut regretter qu'ils n'en aient pas tiré la conclusion que la perte dans la transmission du message par l'intermédiaire de l'interprète, auditeur attentif et intelligent, est susceptible dans ce cas d'être inférieure à la perte accompagnant la transmission directe.

Sur le plan théorique, on peut reprocher à ce livre une optique trop «behavioriste», les mots en langue de départ étant présentés comme appelant des mots en langue d'arrivée, plutôt que comme matière première pour une interprétation sémantique suivie de la réexpression du message: dans le chapitre sur la prise de notes en consécutive, les auteurs conseillent de prendre des notes en langue d'arrivée, ce qui permet de «lire les mots» au stade de la réexpression et «de ne plus avoir d'encodage à faire»; plus loin, dans le chapitre consacré à l'apprentissage de la simultanée, ils recommandent des exercices de répétition, puis de «traduction» de mots isolés lus à des intervalles de plus en plus brefs. Les auteurs croient-ils vraiment au transcodage? Peut-être pas, car ils préconisent par ailleurs l'utilisation de dessins dans les notes de consécutive et une interprétation non littérale et intelligente, mais l'impression d'une «interprétationréflexe linguistique» qui se dégage des passages cités plus haut a de quoi susciter des interrogations.

Une autre erreur, que l'on retrouve d'ailleurs dans de nombreux autres livres et articles sur l'interprétation au Japon, consiste à considérer que si le japonais est une langue à part, les langues occidentales, et notamment le français et l'anglais, sont tellement proches que le transcodage entre elles est possible. Pour illustrer cette affirmation, une phrase japonaise est présentée avec sa traduction anglaise, ainsi que sa traduction française, dont la structure est effectivement proche de la structure de l'anglais et très différente de celle du japonais :

Le Président remercie l'Association pour leurs efforts à préparer la conférence malgré toutes les difficultés à surmonter et les interprètes pour leurs services rendus (p. 43).

En lisant cet exemple, et compte tenu de l'existence d'exemples analogues dans d'autres livres, on est frappé de l'amateurisme avec lequel les Japonais traitent parfois les 
langues occidentales autres que l'anglais, peut-être en raison de leur position éminemment minoritaire au Japon.

Par contre, Tsuyaku présente des idées fort intéressantes pour les Occidentaux sur la langue japonaise et la communication chez les Japonais.

On y trouve entre autres l'observation suivante, que font également d'autres livres et articles sur la psychologie et la sociologie de la communication au Japon: il y aurait bien peu de véritables discussions dans les conférences japonaises, car les questions importantes seraient réglées par l'autorité que donne l'âge ou la position sociale plutôt que par des considérations objectives, et les conflits éventuels seraient résolus préalablement dans les coulisses, ce qui fait que les conférences serviraient essentiellement à officialiser ce qui aurait déjà fait l'objet d'un accord entre les parties.

Selon une deuxième idée soulignée par ce livre et représentative d'un courant de pensée dominant au Japon, les Japonais répugnent à être trop «clairs» et trop tranchants dans leurs paroles, d'où un discours plutôt vague et des procédés de communication accordant une part importante au non-dit, ce qui d'une part met mal à l'aise les Occidentaux, qui préfèrent les situations explicites, et d'autre part pose problème pour les interprètes travaillant vers les langues occidentales.

Sur le plan linguistique, les auteurs soulignent la difficulté qu'engendre le grand nombre d'homonymies dans le vocabulaire japonais, ainsi que l'intense effort de mémoire à court terme en simultanée qui est la conséquence pratique des différences de structure entre le japonais et l'anglais. Ils conseillent une stratégie d'interprétation fondée sur l'anticipation et la segmentation des phrases en plus petites unités de sens, deux procédés permettant d'alléger cet effort de mémoire (on notera que le procédé de segmentation est préconisé depuis de nombreuses années par Gérard ILG à propos de l'interprétation de l'allemand vers le français, qui pose des problèmes de mémoire semblables à ceux du japonais).

Les conseils méthodologiques et didactiques des trois auteurs sont proches de ceux que l'on trouve chez les auteurs occidentaux: ils soulignent entre autres la nécessité pour l'interprète d'acquérir une excellente maittrise de la langue maternelle, de bonnes connaissances générales et l'habitude de s'exprimer avec logique et clarté, et conseillent la lecture de textes juridiques à ce propos.

Par rapport aux ouvrages publiés sur l'interprétation à la même époque en Europe, outre la grande richesse de ses idées, ce livre a surtout le mérite de ne pas détourner les yeux des différentes difficultés techniques et humaines de l'interprétation et de leurs conséquences. Les auteurs n'hésitent pas à souligner qu'en simultanée, les délégués peuvent être favorablement impressionnés par les paroles d'un interprète qui ne travaille pas nécessairement bien mais dont le débit est rapide, ce qui permet de faire «passer» une qualité de travail moindre, ou de déconseiller des demandes d'explications trop fréquentes à l'orateur, celles-ci risquant d'entraîner une perte de confiance à l'égard de l'interprète. De même sont mentionnés le statut social trop bas des interprètes, leur rémunération, leurs relations avec les clients qu'il faut par exemple éviter d'éclipser par une présence trop forte, l'attitude à prendre quand on sert de bouc émissaire ou quand on est injustement accusé d'avoir mal interprété, les attitudes corporelles des interprètes, leur comportement en cabine, la frustration de l'interprète qui n'a pas d'autre activité professionnelle.

Tsuyaku n'est pas un traité théorique, ni un manuel d'apprentissage. Il contient toutefois tous les éléments de base dont peut avoir besoin un enseignant pour faire son cours d'interprétation, en développant au besoin certaines idées. C'est pourquoi en dépit de ses faiblesses théoriques, ce livre a une qualité incontestable, surtout si l'on tient compte de la date de sa publication. 
Étonné par le fait que ce livre, qui avait connu un si grand succès lors de sa publication, n'ait pas été réédité depuis, nous avons posé la question au responsable du service compétent dans la maison d'édition concernée (qui fait partie de l'organe national de radio et de télévision NHK); d'après celui-ci, la vague de popularité de l'interprétation déclenchée par «Apollo» était de forte amplitude, mais de brève durée, et ne justifiait pas la réédition du livre. À méditer...

Malheureusement, écrit en japonais, Tsuyaku est totalement inconnu au sein de la communauté des interprètes occidentaux. S'il avait été traduit et diffusé en Europe, peutêtre sa démarche si différente de celle des auteurs occidentaux aurait-elle pu les inspirer, et notamment servir de matière à réflexion pour les chercheurs, qui, à de rares exceptions près, ont suivi une voie peut-être trop abstraite et absolue, et par là méthodologiquement et pratiquement moins féconde qu'elle aurait pu l'être.

DANIEL GILE

Note

L'auteur remercie la Fondation du Japon qui, dans le cadre d'une bourse de recherche à Tokyo, lui a permis d'étudier de plus près les activités de traduction et d'interprétation au Japon, et de tenter de les faire connaître en Occident à travers les pages des revues spécialisées. 